\title{
Transposition
}

Musique et Sciences Sociales

\section{Le prix de la musique}

\section{David Christoffel, Ouvrez la tête (ma thèse sur Satie)}

Paris, Éditions MF, 2016.

\section{Julien Labia}

\section{CpenEdition}

\section{Journals}

Édition électronique

URL : http://journals.openedition.org/transposition/2657

DOI : 10.4000/transposition.2657

ISSN : 2110-6134

Éditeur

CRAL - Centre de recherche sur les arts et le langage

Référence électronique

Julien Labia, «David Christoffel, Ouvrez la tête (ma thèse sur Satie) », Transposition [En ligne], 7 | 2018,

mis en ligne le 15 septembre 2018, consulté le 25 septembre 2020. URL : http://

journals.openedition.org/transposition/2657 ; DOI : https://doi.org/10.4000/transposition.2657

Ce document a été généré automatiquement le 25 septembre 2020.

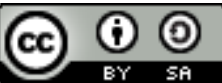

La revue Transposition est mise à disposition selon les termes de la Licence Creative Commons Attribution - Partage dans les Mêmes Conditions 4.0 International. 


\section{David Christoffel, Ouvrez la tête (ma thèse sur Satie)}

Paris, Éditions MF, 2016.

Julien Labia

\section{RÉFÉRENCE}

David Christoffel, Ouvrez la tête (ma thèse sur Satie), Paris, Éditions MF, 2016.

1 Y aurait-il enfin un "cas " Satie? On peut s'étonner du peu d'intérêt du système planétaire universitaire gravitant autour de la musique pour un compositeur qu'on trouve, somme toute, plus souvent cité et analysé dans des livres portant sur les arts plastiques ou sur l'art au $\mathrm{xx}^{\mathrm{e}}$ siècle en général. Il est indéniable que son Cuvre protéiforme paraisse fuyante, moins soucieuse d'être comprise que d'échapper à la fois aux méthodes courantes d'analyse et à un certain esprit de sérieux. On rappellera bien sûr combien fut précieux, avant ce livre, le travail de Vladimir Jankélévitch sur le " dégrisement» dans la musique d'Erik Satie, sans doute à réévaluer aujourd'hui ${ }^{1}$. Le fait qu'il appartienne à une autre époque de la philosophie de la musique au moins autant qu'à un âge révolu de la «musicographie " n'en abolit certainement pas la valeur presque pionnière. Usant d'autres chemins et soucieux de précision, ce nouvel ouvrage riche et charpenté ouvre des pistes fortes et inédites sur l'œuvre aux cent portes du compositeur d'Arcueil.

2 Aborder l'œuvre de Satie pose à l'évidence d'importantes questions de méthode car il semble falloir respecter, en plus de la lettre d'une œuvre écrite - le livre de David Christoffel montre qu'on y rencontre l'écrit sous toutes ses formes - un certain esprit. C'est pourquoi l'auteur prend soin de respecter l'invention déroutante de l'œuvre du maître d'Arcueil en ajoutant à son livre un chapitre dont la poésie consonne avec celle de Satie sans chercher pourtant à l'imiter: des remarques détachées nées à l'écoute d'une de ces "performances" contemporaines appelées par la musique de Satie, intitulées Avantages à écouter Vexations 840 fois (p. 329-337). Citons ici quelques-unes des 
variations poétiques nées au fil cette œuvre "minimale " destinée à être jouée en boucle : " on en connaît qui pensent savoir ce qui est important - et qui ne sont pas là " (p. 334), «c'est très varié, ce qu'on peut ressentir - plus encore que ce qu'on peut faire» (p.335), "encore un privilège que les notoires n'auront pas» (p.333), ou l'héraclitéen « flotter ne veut pas forcément dire se mouiller » (p. 333).

L'objet central du livre, bien sérieux, est clairement indiqué par le titre de la thèse de l'auteur, point de départ justement rappelé : «Les mentions verbales sur les partitions pour piano seul d'Erik Satie ». De la vocation de ce travail initial, que l'auteur désigne avec autodérision comme celui d'un "musicologue justicier », est donc né ce livre au titre énigmatique: Ouvrez la tête ( $m a$ thèse sur Satie). Sous ce possessif mis entre parenthèses, on croit reconnaître un peu d'Alfred Jarry (cité d'ailleurs p. 294), mettant dans la bouche d'Ubu roi : «Qu'on me donne MA liste de MES biens!». Il y a pourtant dans l'ouvrage largement autant de sérieux que d'indispensable stratégie. La démarche du livre relève en effet tout autant de la Philosophie (revendiquée p.19) de la Communication (d'où sa référence à la question du perlocutoire, p. 13 ou à Jakobson, p. 181, entre autres) que de la Musicologie, dont l'auteur se demande avec humour si elle n'a pas parfois cherché à se protéger de Satie en l'excluant au prix de tant d'efforts. La justification de cette concentration sur les mentions verbales est ainsi double, puisqu'il s'agit (1) d'éviter d'en faire un simple ajout sur une musique souvent rétive aux critères du jugement de valeur musicologique et (2) de se concentrer ainsi sur un aspect totalement original de l'œuvre de Satie, ces inscriptions au statut indéfinissable dont " ouvrez la tête ", fournissant le titre, est un exemple. Car il faut bien trouver un accès à cette œuvre, chérie des pianistes amateurs et des professionnels qui en multiplient les intégrales, aimée du grand public et pourtant bannie des salles de concert (fait unique, elle n'y est même pas jouée par les célébrités qui l'enregistrent). Voici donc le pari du livre : montrer, si l'on veut bien nous passer l'expression, que ces mentions font de l'œuvre de Satie une "Thèbes aux cent portes ", mais qu'elles jouent aussi bien le rôle de portes d'entrée que de portes de sortie. On comprend alors ainsi le possessif «ma thèse » : il s'agit de parcourir l'‘Euvre grâce à ces mentions verbales qui tiennent parfois du sphinx ou de l'oracle, non de prétendre l'épuiser ou dresser ce cadastre qu'elle interdit visiblement. Cette idée d'un parcours en mouvement s'illustre par les titres retenus pour les sections du livre, qui sont autant de verbes d'action suggérant, outre la musique, le travail du matériau et parfois même celui du graphiste : présenter, s'étirer, mettre en page, phraser, déponctuer, scénariser, poétiser, ironiser, s'adresser, contextualiser, méta-fictionnaliser. À la multitude de ces gestes "poétisant l'espace partitionnel " (p. 321) car refusant la "dramatisation conventionnelle du concert» correspond in fine un chapitre de « conclusions » elles aussi au pluriel. On retient d'elles en particulier l'idée centrale d'un geste de " précarisation » de l'œuvre musicale chez Satie (p. 326), qui referme sans doute lucidement la boucle de l'incompréhension du compositeur d'où partait l'ouvrage. Nous montrer comment y avoir accès, c'est en effet aussi nous montrer comment on a pu se l'interdire.

Cette logique d'entrées-sorties ne doit pourtant pas donner l'idée que l'œuvre de Satie manquerait de consistance, abordée ainsi sous l'angle de la question poétique comme un simple lieu de passage. L'auteur révèle en effet dans son livre le rôle d'opérateur de complexité assumé par ces mentions verbales, démultipliant le sens de la musique au lieu de l'affaiblir. Citons-en quelques-unes pour le lecteur non-initié, non sans préciser que l'ouvrage, lui, propose de les classifier - souplement - en construisant leur périodicité : «Dans le creux de l'estomac ${ }^{2} »$; «VIF (sans trop) ${ }^{3} » ;$ «Dans une grande 
bonté ${ }^{4}$; « Courageusement facile et complaisamment solitaire ${ }^{5}$ »; « Ne changez pas de couleurs ${ }^{6} »$; «La basse liée, n'est-ce pas ${ }^{7}$ ?»); «En-dedans » puis «Véritablement ${ }^{8} »$; ou encore cette citation littérale, référence comprise: «Nos vieilles mœurs interdisaient au jeune homme pubère de se montrer nu dans le bain, et la pudeur jetait ainsi de profondes racines dans les âmes (Cicéron, De la République, traduction Victor Poupin) $)^{9}$ ».

5 Rejetant aussi bien l'idée d'un Satie plaisantin que l'approche psychologiste y voyant une simple maniaquerie personnelle (p. 22-23), l'auteur aborde ces textes parallèles à la musique comme d'authentiques paramètres de la création et non comme des additions secondaires ou marginales. Il distingue ainsi soigneusement la fonction des indications plus traditionnelles figurant sur les partitions d'orchestre de Satie de celles qui se trouvent sur les partitions pour piano, son objet d'étude propre (p. 33). De nombreuses fonctions des mentions verbales sont évoquées, de la « confusion entre prescription sur le jeu et description du déroulement " naissant d'un simple «Bien » sur une partition (p. 304) à une manière de remuer « entre le compositeur et l'interprète des enjeux qui ne regardent pas, traditionnellement, les rapports entre l'auteur et l'exécutant " (p. 309), en passant par une « remise en question des facteurs d'écoute traditionnels [quand ces mentions] ne sont pas directement adressées à l'auditeur » (p. 181).

6 Le travail proprement historique de l'auteur consiste en particulier à nous livrer une documentation faite de trois dossiers différents, s'attachant sur son objet principal (1) à établir l'évolution de la pratique de la mention verbale chez Satie, (2) à l'éclairer d'importantes mises en perspectives avec d'autres types d'écriture de partitions pour piano (Massenet, notamment, ou Saint-Saëns, dont on redécouvre actuellement la musique pour piano) mais aussi (3) à mettre en parallèle l'écriture verbale de Satie avec l'activité poétique de son temps.

7 Les deux premiers gestes ou deux premiers chapitres (" présenter » puis « s'étirer ») insistent sur l'importance du corps du musicien, enjeu de notations faisant de la partition un objet d'étude (" partologique », dit l'auteur) à part entière. Il s'agira donc à partir du chapitre suivant ("phraser», chap.4) de voir comment indications et musique peuvent dialoguer au bénéfice de l'œuvre écrite. La section suivante, " déponctuer » (chap. 5), tire parti du credo de refuser le « biographisme » pour insister avec raison sur ce qui se trouve objectivement écrit. Et pourtant, l'auteur se garde bien de ne faire de la partition de Satie que des notes inscrites dans une page de poésie : il faut bien que cela reste une partition, quitte à la "scénariser » (chap. 6). Le "poétiser " propre au geste de Satie notamment à partir de 1913 s'avère si multiple qu'on a trop souvent privilégié, en effet, une approche à partir du cliché réversible de la catégorie littéraire de l'ironie plutôt qu'à partir de celle, musicale, de la polyphonie (" poétiser ", p. 193). « Est-ce qu'au fond Satie a vraiment plaisanté ? : : s'appuyant sur le propos d'un pionnier de la défense de la musique de Satie, le pianiste français Aldo Ciccolini, David Christoffel distingue justement la plaisanterie d'un «ironiser» (chap. 8) propre au compositeur, supposant qu'on maintienne soigneusement ce qu'il appelle la « solidarité contrapuntique » (p. 223) entre les mentions verbales et la partition. La réflexion de l'auteur s'engage sur un terrain directement philosophique pour analyser cet humour, Hegel, Bergson et Barthes à l'appui (p. 226 sq.) : l'humour fait-il nécessairement appel à l'hétérogénéité, est-il interdit à la "musique seule »? Il devient alors possible d'établir que ces textes énigmatiques de Satie ne relèvent pas d'un ornemental surajouté (p. 258) et donc inessentiel voire superflu. Les deux gestes ultimes, « contextualiser » (chap. 10) 
et "méta-fictionnaliser» (chap.11) renvoient à la volonté propre à Satie de nous échapper, compliquant mais ouvrant aussi à une lecture du compositeur au regard de son temps. On reconstitue alors au fil de ces analyses la manière dont Satie défit luimême tout «biographisme ", en s'attachant précisément au personnage réel que fut le musicien d'Arcueil. Lorsqu'il souligne que Satie écrit « une musique de représentation qui, affirmant l'inégalité de la relation du représentant (en fait seul en scène) et du représenté (inaccessible), défie toute philosophie de la représentation» (p. 319-320), David Christoffel cite judicieusement Jankélévitch établissant un rapprochement entre Satie et Stravinsky pour qui « l'expression n'a jamais été la propriété immanente de la musique ». Ce croisement avec un autre compositeur de ballets, capable d'affirmer à la fois que la musique est « impuissante à exprimer quoi que ce soit » et de se scandaliser paradoxalement face au Sacre orgiaque de Maurice Béjart parce qu'il n'y avait « pas de sexe » dans l'original, laisse rêveur. On peut espérer maintenant qu'à la suite de ces travaux nous soit un jour offert un livre sur cette autre grande méconnue, l'Æuvre d'orchestre de Satie. Mais en montrant les efforts accomplis à la fois, négativement, pour rejeter la musique de Satie et, positivement, pour en ouvrir ici les sens multiples, ce livre nous assure d'une chose: le vent favorable ou défavorable nécessaire pour mouvoir les cent portes d'entrée ou de sortie de cette đEuvre qui aime à la fois se montrer et se cacher, doit être plus qu'un simple courant d'air. À sa lecture, on comprend ce que nous gagnerions à voir enfin le vent tourner en faveur de Satie.

\section{NOTES}

1. JANKÉLÉVITCH Vladimir, La Musique et les heures, Paris, Seuil, 1988.

2. $2^{\text {de }}$ des Valses distinguées du précieux dégoûté.

3. «Sévère réprimande » des Véritables Préludes Flasques (pour un chien).

4. Gnossienne $\mathrm{n}^{\circ} 2$.

5. « L'Initiation », prélude du $2^{\mathrm{e}}$ acte de la Wagnérie Kaldéenne Le Fils des Étoiles.

6. « Le Water-Chute » des Sports et divertissements.

7. «Idylle » des Avant-dernières pensées.

8. «Commune qui mundi nefas » de la Messe des Pauvres.

9. «Son binocle », $2^{\mathrm{de}}$ des Valses distinguées du précieux dégoûté.

\section{AUTEURS}

\section{JULIEN LABIA}

Professeur agrégé de Philosophie en Classes préparatoires, Julien Labia est docteur de l'Université Paris-Sorbonne. Il a enseigné six ans en qualité d'AM puis d'ATER dans cette même 
université et a été chargé de cours à l'Université de Bourgogne. Ancien post-doctorant des universités Sorbonne-Nouvelle (2013) et Humboldt de Berlin (2014), il a été boursier de la Fritz Thyssen Stiftung (Fondation Maison des Sciences de l'Homme, programme Marie Curie). Membre du jury de l'Agrégation d'Arts appliqués, il est depuis sa création le codirecteur (avec Antonia Soulez) de la collection « Musique \& Philosophie » des éditions Delatour-France (neuf volumes parus depuis 2012 : http://www.editions-delatour.com/fr/27-musique-philosophie). Ses publications portent sur la philosophie de la musique et la critique musicale, et, plus récemment, sur les musiques du monde. Traducteur de l'Esthétique de la musique de Carl Dahlhaus (Vrin, 2015) et de Pensée des sons, thèmes de philosophie de la musique, d'Alessandro Bertinetto (Delatour, 2017), il est par ailleurs l'intervenant du programme « Philo-Musique » de la Philharmonie de Paris, qu'il a conçu (https://fr.calameo.com/read/0025459523485310b6147). https://www.linkedin.com/in/ julien-labia-21764369 